\title{
Renal safety in pediatric imaging: randomized, double-blind phase IV clinical trial of iobitridol 300 versus iodixanol 270 in multidetector CT
}

\author{
Martin Zo'o • Marcus Hoermann • Csilla Balassy • \\ Francis Brunelle • Robin Azoulay • Danièle Pariente • \\ Michel Panuel • Patrick Le Dosseur
}

Received: 17 August 2010 /Revised: 11 February 2011 / Accepted: 4 April 2011 /Published online: 29 June 2011

(C) The Author(s) 2011. This article is published with open access at Springerlink.com

\begin{abstract}
Background It is debated whether iso-osmolar and lowosmolar contrast media are associated with different incidences of contrast medium-induced nephropathy (CIN) in patients with renal insufficiency.

Objective To compare the incidence of CIN in children undergoing contrast-enhanced multidetector computer tomography (MDCT) with intravenous injection of lowosmolar (iobitridol, Xenetix ${ }^{\circledR} 300$ ) or an iso-osmolar (iodixanol, Visipaque ${ }^{\circledR} 270$ ) iodinated contrast medium. Materials and methods One hundred forty-six children with normal renal function were included in this multicenter trial and underwent contrast-enhanced MDCT. The primary
\end{abstract}

M. Zo'o $(\varangle) \cdot$ P. Le Dosseur

Radiology Department, CHU Charles Nicolle,

13 rue Cesar Franck,

Rouen 76000, France

e-mail: zoo_ci@yahoo.fr

M. Hoermann $\cdot$ C. Balassy

Radiology Department, AKH,

Vienna, Austria

F. Brunelle

Radiopediatry Department, Necker Hospital,

Paris, France

R. Azoulay

Radiopediatry Department, Robert Debré Hospital,

Paris, France

D. Pariente

Radiopediatry Department, Kremlin-Bicêtre Hospital,

Kremlin-Bicêtre, France

M. Panuel

Medical Imaging Department, Nord Hospital,

Marseille, France endpoint was the relative change in creatinine clearance from $48 \mathrm{~h}$ pre- to $72 \mathrm{~h}$ postcontrast medium administration using a noninferiority analysis in the intent-to-treat (ITT, $n=128)$ and per protocol $(n=68)$ populations. Secondary endpoints were incidence of CIN, global image quality, diagnostic efficacy and clinical safety.

Results In the ITT population, the noninferiority of iobitridol over iodixanol was demonstrated. CIN incidence was $4.8 \%$ (three cases) with iobitridol and $10.6 \%$ (seven cases) with iodixanol (not significant). No statistically significant differences were observed for the secondary endpoints.

Conclusion Comparable satisfactory safety profiles were confirmed for both contrast media, with no significant difference in the incidence of CIN in children with normal renal function.

Keywords Contrast medium-induced nephropathy . Multidetector computer tomography · Iobitridol · Iodixanol

\section{Introduction}

The use of iodinated contrast media (CM) has increased over the past decades and is a well-known cause of acute renal failure, especially in patients with impaired renal function and/or in those with diabetes mellitus [1-3]. Iodinated contrast medium-induced nephropathy (CIN) is a potentially serious complication of diagnostic or interventional procedures leading to dialysis, prolonged hospitalization, increased morbidity and mortality [4].

Iodinated $\mathrm{CM}$ are either ionic or nonionic and vary in their osmolality relative to plasma. It is now widely acknowledged that high-osmolality $\mathrm{CM}$ (ionic) are more 
nephrotoxic than low-osmolality contrast media (LOCM), ionic or nonionic, in at-risk patients [5]. However, whether there are differences in safety among the various $\mathrm{CM}$, particularly with regard to nephrotoxicity, remains controversial. Many clinical studies and meta-analyses have compared the safety of the various $\mathrm{CM}$; however, depending on the patient population and study design, results are controversial [6-13].

$\mathrm{CIN}$ is generally characterised by an increase in serum creatinine of more than $25 \%$ or $0.5 \mathrm{mg} / \mathrm{dl}(44 \mu \mathrm{mol} / \mathrm{l})$ over baseline, occurring within 3 days of CM administration. Serum creatinine is a relatively crude measure of glomerular filtration rate, and it has been suggested that creatinine clearance should be used to identify at-risk patients, as it provides better indication of renal function $[14,15]$.

The incidence of CIN is low (around 5\%) in patients without risk factors, but is increased (up to $50 \%$ ) in patients with multiple risk factors [16]. These risk factors include contrast dose, contrast osmolality, previous $\mathrm{CM}$ injection and route of administration of CM [17]. The risk of CIN is estimated to be more than twofold lower when administered intravenously rather than intra-arterially $[14,18]$.

Few clinical studies have compared the renal effects of intravenous administration of a low osmolar and an isoosmolar contrast agent. There are very few studies of the effects in children in particular [19, 20].

Whether the use of an isotonic contrast agent confers any additional benefit in children compared with a low-osmolar agent is unclear. Therefore, the purpose of this randomized phase IV multicenter study was to compare the incidence of $\mathrm{CIN}$ in children undergoing enhanced multidetector computer tomography (MDCT) after intravenous administration of a low-osmolar (iobitridol) or an iso-osmolar (iodixanol) contrast agent.

\section{Materials and methods}

Study conduct and patient enrollment conditions

The study was approved by the Institutional Review Board of each participating center and performed according to good clinical practice standards. Informed written consent was obtained from each patient and/or patient's parents before any study-specific procedures were performed.

Children between 1 and 16 years of age with normal renal function (creatinine clearance $>60 \mathrm{ml} / \mathrm{min} / 1.73 \mathrm{~m}^{2}$, calculated by the Schwartz's formula [21]), scheduled to undergo a clinically indicated contrast-enhanced MDCT examination were considered eligible for inclusion. Patients were excluded from the trial in case of pregnancy or known hypersensitivity to iodine-containing compounds. Patients were also excluded if they had received any iodinated contrast agent within 7 days before the administration of the investigational product, if they were scheduled to receive an iodinated contrast agent within $72 \mathrm{~h}$ after administration of the investigational product, if they received any nephrotoxic medication (chemotherapeutic agents, diuretics or biguanide), or if they had any surgery planned within $72 \mathrm{~h}$ after the administration of the contrast agent.

After obtaining written informed consent and verifying all selection criteria, patients included in the study were then allocated an identification number corresponding to their chronological rank of inclusion. Patients were assigned randomly to intravenous administration of the LOCM iobitridol $\left(695 \mathrm{mOsm} / \mathrm{kg} \mathrm{H}_{2} \mathrm{O}\right)$ or the IOCM iodixanol $\left(290 \mathrm{mOsm} / \mathrm{kg} \mathrm{H} \mathrm{H}_{2} \mathrm{O}\right)$. LOCM are defined as $\mathrm{CM}$ with an osmolality two to three times that of normal human blood at an iodine concentration around $300 \mathrm{mg} / \mathrm{ml}$. IOCM are defined as CM that are iso-osmolar to blood at an iodine concentration around $320 \mathrm{mg} / \mathrm{ml}$. All groups and individuals associated with the study remained blinded to the type of contrast medium until the database was locked and the data analyzed. The randomization list was stratified by center. The MDCT images were assessed subjectively on-site by one experienced reader at each participating site. If needed, patients between 1 and 6 years received hydroxyzine for sedation ( $2 \mathrm{mg} / \mathrm{kg}$ body weight). Preprocedure hydration was not implemented in this study.

\section{Contrast medium administration}

To ensure blinding at the investigational sites, a third-party operator dispensed the drugs, managed the preparation, dispensation and accountability of the investigational agents, as per code assignment. The sole responsibility of all operators who dispensed the drugs was to preserve the blinding, and therefore they did not participate in any of the study assessments.

Contrast medium (iobitridol, Xenetix ${ }^{\circledR}$ 300, Guerbet Laboratories, Roissy, France or iodixanol, Visipaque ${ }^{\circledR} 270$, GE Healthcare SA) was administered by intravenous injection according to a total volume depending on the patient's weight. All scans were performed following administration of $2 \mathrm{ml} / \mathrm{kg}$ body weight, maximum $100 \mathrm{ml}$, of contrast medium. The first part of the study applied a maximum volume of $50 \mathrm{ml}$.

Evaluation of primary and secondary endpoints

The primary endpoint was the relative change [22] in creatinine clearance from $48 \mathrm{~h}$ before to $72 \mathrm{~h}$ after contrast medium administration using a noninferiority analysis. 
The secondary endpoints were the incidence of CIN (defined as more than $25 \%$ reduction in creatinine clearance), global image quality on a 3-point scale (poor: low and inhomogeneous enhancement of target structures, moderate: little, but still homogeneous enhancement of target structures, good: adequate, almost completely homogeneous signal enhancement of target structures) and diagnosis efficacy on a 3-point scale (impossible: not providing enough information, difficult: not providing all the expected information, easy: providing the expected information). Moreover, the safety profile (vital signs and adverse events) of both contrast agents was also recorded. Any event from inclusion until the end of the 10-day follow-up period was collected and reported.

We evaluated the causality between the investigational product and /or concomitant therapy and the adverse event. The imputability of any reactions to the contrast agent was excluded if no causal relationship existed between the study drug and the event and an obvious alternative cause existed (e.g., the patient's underlying medical condition or concomitant therapy).

\section{Statistical analysis}

Assuming that the relative variation of the creatinine clearance from baseline is around $0.10(10 \%)$ on average for both contrast agents with a 0.10 standard deviation, a sample of 128 patients was considered sufficient for $80 \%$ power and $2.5 \%$ type- 1 error to ensure that if the difference between both products would not exceed -0.05 in disfavor of iobitridol, the noninferiority of iobitridol would be demonstrated over iodixanol. Assuming a 10\% dropout, the sample size was extended to 142 patients to reach the main study objective.

Creatinine clearance was calculated in both groups before and after injection of contrast agents and the relative variation of these measures was computed and compared in each group. The noninferiority analysis on the primary endpoint was tested using the noncentral Student's $t$-test with a 2-sided type-1 error set at 0.05 . The noninferiority of iobitridol over iodixanol was established if the observed difference between groups differed from the noninferiority clinical limit set at -0.05 in the study. This margin was considered by the study investigators as clinically sufficiently low to conclude a nondifference between the contrast agents.

Descriptive statistics were used for demography and baseline characteristics. The statistical test used for analyzing $\mathrm{CIN}$ incidence, image quality and diagnostic efficacy was the Wald Chi-2 test using a generalized linear model. The $P$-value for the main effect (difference between products) was adjusted on centers, mainly to take into account the stratification of the randomization by centre.
Statistical analyses were conducted using the software SAS version 9.1 (SAS Institute, Cary, N.C.).

The intent-to-treat (ITT) population refers to patients receiving at least one injection of contrast agent, and presenting with a valid creatinine clearance variation. The findings in this population were used for the primary and secondary criteria analyses. The per protocol (PP) population includes patients where there was no protocol deviation or violation. This population was also included in the primary and secondary criteria analyses. The safety population included all patients who received at least one dose of the study product and was used for the safety analyses.

\section{Results}

Patients

Between May 2006 and March 2009, 146 children with normal kidney function scheduled to undergo clinically indicated MDCT were recruited. One patient did not receive the product, hence, 145 children were randomized to receive iobitridol $300(n=74)$ or iodixanol $(n=71)$, and were all eligible for the safety analysis.

As shown in Table 1, the two treatment groups were well-balanced with regard to demographic and baseline data. None of the patients had renal insufficiency. The most common indication was the "others" category, with the largest sub categories being liver transplant assessment, headache and Crohn disease.

Among the 146 children enrolled in the study, 18 were excluded from the ITT analysis, making 128 children eligible for the ITT population. The main reason for excluding children from ITT analysis was missing creatinine measurement.

From the ITT population, 68 children were free of major protocol deviations and were therefore eligible for the perprotocol analysis. The main reasons for excluding children from PP analysis were creatinine measurement out of time frame ( $48 \mathrm{~h}$ before injection or $72 \pm 24 \mathrm{~h}$ after injection), contrast medium dose out of range $(2 \mathrm{ml} / \mathrm{kg} \pm 10 \%)$ and code breaking.

\section{Primary endpoint}

In the ITT population $(n=128)$, the mean difference (iobitridol-iodixanol) in creatinine clearance variation from baseline was $-0.12 \%(P=0.042)$, which was significantly different from the noninferiority margin $(-0.05)$, demonstrating the noninferiority of iobitridol over iodixanol. The results were consistent in the per-protocol population (68 children), with a mean difference of $2.2 \%(P=0.027$; Table 2$)$. 
Table 1 Demographic and baseline characteristics, indications for multidetector computer tomography (MDCT) and patient condition. BMI Body mass index

\begin{tabular}{|c|c|c|c|}
\hline & Iobitridol $300(n=74)$ & Iodixanol $270(n=71)$ & Total $(n=145)$ \\
\hline \multicolumn{4}{|l|}{ Baseline characteristics } \\
\hline Age (years) mean $\pm \mathrm{SD}$, (min-max) & $8.7 \pm 4.8(1.0-16.0)$ & $8.1 \pm 4.7(0.0-16.0)$ & $8.4 \pm 4.7(0.0-16.0)$ \\
\hline Male & $43(58.1 \%)$ & $43(60.6 \%)$ & $86(59.3 \%)$ \\
\hline Female & $31(41.9 \%)$ & $28(39.4 \%)$ & $59(40.7 \%)$ \\
\hline BMI $\left(\mathrm{kg} \mathrm{m}^{-2}\right)$ mean $\pm \mathrm{SD},(\min -\max )$ & $17.7 \pm 3.7(12.7-33.6)$ & $17.4 \pm 3.2(11.8-25.7)$ & $17.6 \pm 3.4(11.8-33.6)$ \\
\hline $\begin{array}{l}\text { Baseline serum creatinine }(\mu \mathrm{mol} / \mathrm{l}) \text { mean } \pm \mathrm{SD} \text {, } \\
(\min -\max )\end{array}$ & $46.4 \pm 15.8(17.9-84.0)$ & $43.6 \pm 17.1(15.0-89.0)$ & $45.0 \pm 16.5(15.0-89.0)$ \\
\hline $\begin{array}{l}\text { Baseline creatinine clearance }\left(\mathrm{ml} \mathrm{min}{ }^{-1} 1.73 \mathrm{~m}^{-2}\right) \\
\text { mean } \pm \mathrm{SD},(\min -\max )\end{array}$ & $154.7 \pm 44.3(70.8-315.6)$ & $157.9 \pm 44.4(73.5-260.7)$ & $156.4 \pm 44.2(70.8-315.6)$ \\
\hline \multicolumn{4}{|l|}{ Indications for MDCT examination $(\mathrm{N}, \%)$} \\
\hline \multicolumn{4}{|l|}{ Congenital malformation } \\
\hline Cardiac & $15(20.3 \%)$ & $11(15.5 \%)$ & $26(17.9 \%)$ \\
\hline Vascular & $5(6.8 \%)$ & $4(5.6 \%)$ & $9(6.2 \%)$ \\
\hline Others & $3(4.1 \%)$ & $5(7.0 \%)$ & $8(5.5 \%)$ \\
\hline \multicolumn{4}{|l|}{ Tumors } \\
\hline Abdomino-pelvic & $1(1.4 \%)$ & $5(7.0 \%)$ & $6(4.1 \%)$ \\
\hline Cerebral & $1(1.4 \%)$ & $3(4.2 \%)$ & $4(2.8 \%)$ \\
\hline Others & $6(8.1 \%)$ & $3(4.2 \%)$ & $9(6.2 \%)$ \\
\hline Trauma & $6(8.1 \%)$ & $4(5.2 \%)$ & $10(6.9 \%)$ \\
\hline Other $^{\mathrm{a}}$ & $41(55.4 \%)$ & $42(59.2 \%)$ & $83(57.2 \%)$ \\
\hline \multicolumn{4}{|l|}{ Patient disposition } \\
\hline Total number of randomized patients & 74 & 71 & 145 \\
\hline Safety population & $74(100 \%)$ & $71(100 \%)$ & $145(100 \%)$ \\
\hline Intent-to-treat population & $62(83.8 \%)$ & $66(93.0 \%)$ & $128(88.3 \%)$ \\
\hline Per protocol population & $29(39.2 \%)$ & $39(54.9 \%)$ & $68(46.9 \%)$ \\
\hline
\end{tabular}

${ }^{a}$ Mainly liver transplantation, headache and Crohn disease

\section{Secondary endpoints}

\section{CIN incidence}

CIN was observed in $3 / 62$ patients (4.8\%) with iobitridol and $7 / 66$ patients $(10.6 \%)$ with iodixanol, with no statistically significant difference between groups in the ITT population $(P=0.72)$. Similar results were found in the PP population $(P=0.68)$ (Table 3). Characteristics of these patients are detailed in Table 4.

\section{Image quality assessment}

The image quality rated "good" was similar in both groups: 52/62 patients (83.9\%) with iobitridol 300 versus $59 / 66$ patients $(89.4 \%)$ with iodixanol 270 , with no

Table 2 Increase in creatinine clearance at day 3 following contrast medium administration compared to baseline. ITT Intent-to-treat population, $P P$ per protocol population

\begin{tabular}{llll}
\hline $\begin{array}{l}\text { Creatinine clearance } \\
\left(\mathrm{ml} \mathrm{min} \mathrm{m}^{-1} 1.73 \mathrm{~m}^{-2}\right)\end{array}$ & Iobitridol 300 & Iodixanol 270 & $\begin{array}{c}\text { Difference (iobitridol-iodixanol) } \\
(90 \% \text { confidence interval) } P\end{array}$ \\
\hline ITT & $n=62$ & $n=66$ & $-0.12 \%(-0.0481 ; 0.0456) P=0.042$ \\
Mean $(\% \pm \mathrm{SD})$ & $0.011 \pm 0.197$ & $0.019 \pm 0.220$ & \\
Min/max & $-0.387 / 0.953$ & $-0.584 / 0.769$ & $2.2 \%(-0.0405 ; 0.0844) P=0.027$ \\
PP & $n=29$ & $0.018 \pm 0.200$ & \\
Mean $(\% \pm \mathrm{SD})$ & $0.039 \pm 0.222$ & $-0.320 / 0.693$ & \\
Min/max & $-0.237 / 0.953$ & & \\
\hline
\end{tabular}


Table 3 Incidence of contrast medium-induced nephropathy (CIN) (creatinine clearance reduced $>25 \%$ compared to baseline)

\begin{tabular}{llll}
\hline & Iobitridol 300 & Iodixanol 270 & $P^{\mathrm{a}}$ \\
\hline ITT & $n=62$ & $n=66$ & 0.72 \\
& $3(4.8 \%)$ & $7(10.6 \%)$ & \\
PP & $n=29$ & $n=39$ & 0.68 \\
& $0(0 \%)$ & $4(10.3 \%)$ & \\
\hline
\end{tabular}

${ }^{\text {a }}$ Wald Chi-2 test using a generalized linear model. The $P$-value for the main effect (difference between products) was adjusted on centers

statistically significant difference between groups $(P=$ 0.73) (Table 5).

Image quality was judged as "poor" or "moderate" in patients with high BMI, in those who did not receive a sufficient dose, and in the one case with extravasation, in the iobitridol group, when contrast administration had to be suspended.

\section{Diagnostic efficacy}

The diagnostic efficacy rated "easy" was similar in both groups: 56/62 patients (90.3\%) with iobitridol 300 versus $65 /$ 66 patients $(98.5 \%)$ with iodixanol 270 , with no statistically significant difference between groups $(P=0.58)$ (Table 5).

When the diagnostic efficacy was assessed as impossible or difficult, this was related mainly to patients with poor or moderate image quality.

\section{General safety}

The mean volume of contrast agent injected was $54.2 \mathrm{ml}(10$ $100 \mathrm{ml}$ ). The mean amount of iodine administered was slightly higher in the iobitridol 300 group $[16.3 \pm 7.3 \mathrm{~g}(3.0-$ $30.0)]$ compared to the iodixanol 270 group $[14.6 \pm 7.0 \mathrm{~g}$ (3.24-27.0)].

All patients were followed for 10 days after MDCT. A total of 16/74 patients (21.6\%) with iobitridol 300 and 17/ 71 patients $(23.9 \%)$ with iodixanol 270 experienced at least one adverse event. A total of 43 adverse events were observed: $20(46.5 \%)$ with iobitridol 300 (including two pre-injection adverse events) and $23(53.4 \%)$ with iodixanol 270. Most of these were of mild/moderate intensity. Relationship to the products was assumed for $9(45 \%)$ adverse events ( 1 case of pruritis, 3 cases of rash, 1 case of extravasation and 4 cases of increased creatinine) with iobitridol 300 and 12 (52.1\%) adverse events (1 case each of dermatitis, injection site warmth, pyrexia, rash and nausea, 5 cases of increased blood creatinine and 2 cases of vomiting) with iodixanol 270.

A total of nine serious adverse events were reported, five following iobitridol 300 and four following iodixanol 270 . None of these was considered related to the products.

\section{Discussion}

This double-blind, randomized, controlled study performed in children with normal renal function showed that iobitridol 300 was noninferior to iodixanol 270 in terms of relative variation of the creatinine clearance despite a higher iodine load in the iobitridol group.

Many studies have demonstrated that high-osmolar contrast media are more nephrotoxic than new generation, low and iso-osmolar alternatives [3, 23, 24], as also noted in the guidelines from the European Society of Urogenital
Table 4 Characteristics of children with contrast medium-induced nephropathy

\begin{tabular}{|c|c|c|c|c|c|}
\hline Patients & $\begin{array}{l}\text { Gender/ age } \\
\text { (years) }\end{array}$ & $\begin{array}{l}\text { BMI } \\
\left(\mathrm{kg} \mathrm{m}^{-2}\right)\end{array}$ & $\begin{array}{l}\text { Volume } \\
\text { administered } \\
(\mathrm{mL})\end{array}$ & $\begin{array}{l}\text { Relative variation } \\
\text { in creatinine } \\
\text { clearance }(\%)\end{array}$ & Indications for MDCT \\
\hline \multicolumn{6}{|l|}{ Iobitridol } \\
\hline \#1 & Female/11 & 18.3 & 74 & -26 & $\begin{array}{l}\text { Bile-duct atresia with } \\
\text { secondary cirrhosis } \\
\text { complication }\end{array}$ \\
\hline$\# 2$ & Male/4 & 15.1 & 26 & -32 & Neck tumor \\
\hline \#3 & Male/2 & 16.7 & 26 & -39 & Kawasaki disease \\
\hline \multicolumn{6}{|l|}{ Iodixanol } \\
\hline \#4 & Female/12 & 15.6 & 88 & -32 & Liver transplantation \\
\hline \#5 & Male/4 & 16 & 32 & -40 & Chronic osteomyelitis \\
\hline$\# 6$ & Female/7 & 17.2 & 50 & -29 & Cerebral tumor \\
\hline \#7 & Female/12 & 14.3 & 50 & -58 & Cystic fibrosis \\
\hline$\# 8$ & Female/2 & 13.8 & 20 & -28 & Retroperitoneal tumor \\
\hline$\# 9$ & Male/1 & 16.6 & 24 & -29 & Pneumonia \\
\hline$\# 10$ & Female/16 & 22.9 & 70 & -26 & $\begin{array}{l}\text { Pulmonary graft versus } \\
\text { host reaction }\end{array}$ \\
\hline
\end{tabular}


Table 5 Image quality and diagnostic efficacy assessments

\begin{tabular}{|c|c|c|c|}
\hline & $\begin{array}{l}\text { Iobitridol } 300 \\
(n=62)\end{array}$ & $\begin{array}{l}\text { Iodixanol } 270 \\
(n=66)\end{array}$ & $P^{\mathrm{a}}$ \\
\hline \multicolumn{4}{|l|}{ Image quality } \\
\hline Poor/moderate & $10(16.1 \%)$ & $7(10.6 \%)$ & 0.73 \\
\hline Good & $52(83.9 \%)$ & $59(89.4 \%)$ & \\
\hline \multicolumn{4}{|l|}{ Diagnostic efficacy } \\
\hline Impossible/difficult & $6(9.7 \%)$ & $1(1.5 \%)$ & 0.58 \\
\hline Easy & $56(90.3 \%)$ & $65(98.5 \%)$ & \\
\hline
\end{tabular}

${ }^{\text {a }}$ Wald Chi-2 test using a generalized linear model. The $P$-value for the main effect (difference between products) was adjusted on centres

Radiology [25]. Two of the most widely used new contrast media, iodixanol (a nonionic, iso-osmolar dimer) and iobitridol (a nonionic low-osmolar monomer), have consistently been found adequately safe in adults, both in intravenous administration to high-risk patients [26]. However, Chalmers and Jackson found that iodixanol was less nephrotoxic than iohexol (another nonionic lowosmolar monomer), when administered to patients with renal impairment [27]. This superior safety of iodixanol compared to iohexol was also suggested in the NEPHRIC study [13].

In our study including children with normal renal function, iobitridol 300, a low-osmolar contrast agent (695 mOsm/Kg), appeared to be associated with a lower CIN incidence rate $(4.8 \%)$ compared to iodixanol 270 $(10.6 \%)$, an iso-osmolar agent $(290 \mathrm{mOsm} / \mathrm{kg})$. To our knowledge, this is the first study performed in children with normal renal function; therefore, as there is no available data regarding the $\mathrm{CIN}$ incidence on this kind of population, no comparison can be made.

To evaluate whether iodixanol provides a general benefit over other low-osmolar agents in patients with impaired renal function, a number of prospective clinical studies have been conducted recently. The results of these studies showed that the overall benefit of iodixanol over other lowosmolar agents cannot be demonstrated. Briguori and colleagues [10] reported that CIN rates were not significantly different when patients with chronic renal insufficiency were randomized to iodixanol or iobitridol for angiography procedures. In the RECOVER study, iodixanol was compared to the ionic dimer ioxaglate [11]. In this latter study, a significant reduction in the CIN incidence was shown with iodixanol, but only when the two endpoints, serum creatinine increases $\geq 0.5 \mathrm{mg} / \mathrm{dL}$ or $25 \%$ from baseline, were combined. Another recent study, ICON, [28] failed to demonstrate a benefit with iodixanol over ioxaglate in patients with impaired renal function who underwent cardiac angiography. Barrett and colleagues [12] also reported low incidence of CIN in patients with chronic kidney disease randomly assigned to receive iodixanol or iopamidol for contrast-enhanced CT $(2.6 \%$ versus $0 \%$, respectively, $P=0.2)$. The CARE study [29], another and larger study (414 patients), also failed to demonstrate a benefit with iodixanol over iopamidol in renally impaired cardiac angiography patients. Rates of CIN were comparable after using the two agents (12.4\% versus $9.4 \%$, respectively, $P=$ 0.44). In the ACTIVE trial (148 patients), Thomsen and colleagues [30] concluded that the CIN incidence was significantly higher after intravenous administration of iodixanol 320 than iomeprol 400, with an overall rate of CIN in all study groups of 3.4\%. Kuhn and colleagues [14] in the PREDICT study (248 patients) concluded that the overall CIN rate was 5.2\% with iodixanol 320 and iopamidol 370 in patients with both renal impairment and diabetes mellitus who underwent CE-MDCT. Rudnick et al. in the VALOR trial [31] including 299 patients showed that the CIN incidence was $21.8 \%$ with iodixanol and $23.8 \%$ with ioversol $(P=0.78)$, and suggested that the nephrotoxicity associated with iodixanol was not significantly different from that observed with the low-osmolar contrast agent. In a recent meta-analysis by Heinrich et al. [32], a reduction in nephrotoxicity was seen only when iodixanol was compared with iohexol, but no benefit was seen when this IOCM was compared with other LOCM. The results of Alexopoulos et al. [33] showed that no differences in contrast-induced acute kidney injury incidence were apparent among patients receiving nonionic iso-osmolar iodixanol and nonionic lowosmolar contrast agents.

As described above, most of the published reports studied adult patients with pre-existing renal insufficiency and the criteria which identified cases of CIN were not uniform.

Also, the results of our study suggest that differences in $\mathrm{CIN}$ incidence cannot be explained solely by differences in contrast media osmolality [34]. The mechanism of contrastinduced nephropathy is multifactorial and unclear. As described in some studies, factors other than osmolality (such as viscosity, temperature, hydrophilicity) may also contribute to the toxic effect of contrast products [35-37], and perhaps both osmolality and viscosity may also contribute to nephrotoxicity.

The efficacy and safety of iobitridol have been assessed recently in a double-blind, randomized study [38] and a German postmarketing surveillance study involving more than 160,000 patients [39], showing in both studies a diagnostic value and a good safety profile of iobitridol. Regarding the efficacy results in our study, the diagnostic value of MDCT performed in children was shown. The image quality rated "good" was high and similar in both groups, with no statistically significant difference. Although the incidence of patients rated "poor/moderate" was slightly higher with iobitridol $300(16.1 \%)$ than that observed with iodixanol $270(10.6 \%)$, this difference can be explained by 
patients with a high BMI and/or those not receiving a sufficient dose. The diagnostic efficacy rated "easy" was also similar in both groups, with no statistically significant difference.

Finally, the similar and good safety profile of both products was confirmed, and the low incidence of CIN observed in our study suggests that prophylaxis is not required in children when using iobitridol 300 and iodixanol 270.

\section{Conclusion}

This trial suggests that, despite the greater iodine dose, there was no statistically significant difference in the rate of CIN between iobitridol 300 and iodixanol 270 in children with normal renal function. The osmolality of the contrast agent alone probably does not account for the differences in the incidence of CIN. While previous reports have suggested osmolality as a factor in the pathogenesis of $\mathrm{CIN}$ at high osmolalities $(>1,000 \mathrm{mOsm} / \mathrm{kg})$, our results suggest that osmolality in the range of 300 to approximately 700 is not nephrotoxic. Also, for contrast media within this range of osmolality, other characteristics such as viscosity or direct molecular toxicity may play a greater role in the development of CIN. Future prospective clinical trials comparing low and iso-osmolar, as well as low- and high-viscosity $\mathrm{CM}$ in high-risk pediatric patients would be of great interest to explore the role of osmolality and viscosity in CIN.

Acknowledgement This study was supported by Guerbet, France. The authors have no conflicts of interest that are directly relevant to the contents of this study.

Open Access This article is distributed under the terms of the Creative Commons Attribution Noncommercial License, which permits any noncommercial use, distribution and reproduction in any medium, provided the original author(s) and source are credited.

\section{References}

1. Newhouse JH, Kho D, Rao QA et al (2008) Frequency of serum creatinine changes in the absence of iodinated contrast material: implications for studies of contrast nephrotoxicity. AJR 191:376382

2. Turney JH (1996) Acute renal failure: a dangerous condition. JAMA 275:1516-1517

3. Morcos SK, Thomsen HS, Webb JA (1999) Contrast-mediainduced nephrotoxicity: a consensus report. Contrast Media Safety Committee, European Society of Urogenital Radiology (ESUR). Eur Radiol 9:1902-1613

4. Mehran R, Nikolsky E (2006) Contrast-induced nephropathy: definition, epidemiology, and patients at risk. Kidney Int 1000 (suppl):S11-S15
5. Idee JM, Lancelot E, Pines E et al (2004) Prophylaxis of iodinated contrast media-induced nephropathy: a pharmacological point of view. Invest Radiol 39:155-170

6. Sharma S, Kini A (2005) Effect of nonionic radiocontrast agents on the occurrence of contrast-induced nephropathy in patients with mild-moderate chronic renal insufficiency: pooled analysis of the randomized trials. Catheter Cardiovasc Interv 65:386-393

7. McCullough PA, Bertrand ME, Brinker JA et al (2006) A metaanalysis of the renal safety of isosmolar iodixanol compared with low-osmolar contrast media. J Am Coll Cardiol 48:692-699

8. Solomon R, DuMouchel W (2006) Contrast media and nephropathy: findings from systematic analysis and Food and Drug Administration reports of adverse effects. Invest Radiol 41:651-660

9. Liss P, Persson PB, Hansell P et al (2006) Renal failure in 57925 patients undergoing coronary procedures using iso-osmolar or low-osmolar contrast media. Kidney Int 70:1811-1817

10. Briguori C, Colombo A, Airoldi F et al (2005) Nephrotoxicity of low-osmolality versus iso-osmolality contrast agents: impact of $\mathrm{N}$ acetylcysteine. Kidney Int 68:2250-2255

11. Jo SH, Youn TJ, Koo BK et al (2006) Renal toxicity evaluation and comparison between Visipaque (iodixanol) and Hexabric (ioxaglate) in patients with renal insufficiency undergoing coronary angiography. The RECOVER study: a randomized controlled trial. J Am Coll Cardiol 48:924-930

12. Barrett BJ, Katzberg RW, Thomsen HS et al (2006) Contrastinduced nephropathy in patients with chronic kidney disease undergoing computed tomography: a double-blind comparison of iodixanol and iopamidol. Invest Radiol 41:815-821

13. Aspelin P, Aubry P, Fransson SG et al (2003) Nephrotoxic effects in high-risk patients undergoing angiography. $\mathrm{N}$ Engl J Med 348:491-499

14. Kuhn MJ, Chen N, Sahani DV et al (2008) The predict study: a randomized double-blind comparison of contrast-induced nephropathy after low-or isoosmolar contrast agent exposure. AJR 191:151-157

15. Salomon R, Barrett B (2006) Follow-up of patients with contrastinduction-nephropathy. Kidney Int 100(Suppl):S46-S50

16. Reddan D, Laville M, Garovic VD (2009) Contrast-induced nephropathy and its prevention: what do we really know from evidence-based findings? J Nephrol 22:333-351

17. Solomon R (2005) The role of osmolality in the incidence of contrast-induced nephropathy: a systematic review of angiographic contrast media in high-risk patients. Kidney Int 68:2256-2263

18. Katzberg RW, Lamba R (2009) Contrast-induced nephropathy after intravenous administration: fact or fiction. Radiol Clin N Am 47:789-800

19. Hirsch R, Dent C, Pfriem H et al (2007) NGAL is an early predictive biomarker of contrast-induced nephropathy in children. Pediatr Nephrol 22:2089-2095

20. Hui-Stickle S, Brewer ED, Goldstein SL (2005) Pediatric ARF epidemiology at a tertiary care center from 1999 to 2001. Am J Kidney Dis 45:96-101

21. Gruber SJ, Shapiro CJ (2003) Nephropathy induced by contrast medium (letter). N Engl J Med 348:2257

22. Solomon R, Segal A (2008) Defining acute kidney injury: what is the most appropriate metric ? Nat Clin Prac Nephrol 4:208-215

23. Baker CS, Wragg A, Kumar S et al (2003) A rapid protocol for the prevention of contrast-induced renal dysfunction: the RAPID study. J Am Coll Cardiol 41:2114-2118

24. Barrett BJ, Parfrey PS, Vavasour HM et al (1992) Contrast nephropathy in patients with impaired renal function: high versus low osmolar media. Kidney Int 12:3-9

25. Thomsen HS (2003) Guidelines for contrast media from the European Society of Urogenital Radiology. AJR 181:1463-1471

26. Gleeson TG, Bulugahapitiya S (2004) Contrast-induced nephropathy. AJR 183:1673-1689 
27. Chalmers N, Jackson RW (1999) Comparison of iodixanol and iohexol in renal impairment. Br J Radiol 72:701-703

28. Mehran R, Nikolsky E, Kirtane AJ et al (2009) The ICON (Ionic versus non-ionic Contrast to Obviate worsening Nephropathy after angioplasty in chronic renal failure patients) study. J Am Coll Cardiol 2:415-421

29. Salomon RJ, Natarajan K, Doucet $S$ et al (2007) Investigators of the CARE Study. Cardiac Angiography in Renally Impaired Patients (CARE) study: a randomized double-blind trial of contrast-induced nephropathy in patients with chronic kidney disease. Circulation 115:3189

30. Thomson HS, Morcos SK, Erley CM et al, Investigators in the abdominal computed tomography: Iomeron- 400 versus Visipaque320 enhancement (ACTIVE) study (2008) The ACTIVE trial: comparison of the effects on renal functions of iomeprol-400 and iodixanol-320 in patients with chronic kidney disease undergoing abdominal computed tomography. Invest Radiol 43:170-178

31. Rudnick MR, Davidson C, Laskey $W$ et al, VALOR trial investigators (2008) Nephrotoxixity of iodixanol versus ioversol in patients with chronic kidney disease: the Visipaque Angiography/Interventions with Laboratory Outcomes in Renal Insufficiency (VALOR) Trial. Am Heart J 156(4):776-782

32. Heinrich MC, Haberle L, Muller V et al (2009) Nephrotoxicity of iso-osmolar iodixanol compared with nonionic low-osmolar contrast media: meta-analysis of randomized controlled trials. Radiology 250:68-86
33. Alexopoulos E, Spargias K, Kyrzopoulos S et al (2010) Contrastinduced acute kidney injury in patients with renal dysfunction undergoing a coronary procedure and receiving non-ionic lowosmolar versus iso-osmolar contrast media. Am J Med Sci 339:25-30

34. Barrett BJ, Carlisle EL (1993) Meta-analysis of the relative nephrotoxicity of high-and low-osmolality iodinated contrast media. Radiology 188:171-178

35. Rudnick M, Goldfarb S (2003) Pathogenesis of contrastedinduced nephropathy: Experimental and clinical observations with an emphasis on the role of osmolality. Rev Cardiovasc Med 4 (Suppl 5):S28-S33

36. Hizoh I, Strater J, Shick CS et al (1998) Radiocontrast-induced DNA fragmentation of renal tubular cells in vitro: Role of hypertonicity. Nephrol Dial Transplant 13:911-918

37. Hardiek K, Katholi RE, Ramkumar V et al (2001) Proximal tubule cell response to radiographic contrast media. Am J Physiol 280:F61-F70

38. Loewe C, Becker CR, Berletti R et al (2010) 64-slice CT angiography of the abdominal aorta and abdominal arteries: comparison of the diagnostic efficacy of iobitridol $350 \mathrm{mgI} / \mathrm{ml}$ versus iomeprol $400 \mathrm{mgI} / \mathrm{ml}$ in a prospective, randomised, doubleblind multi-centre trial. Eur Radiol 20:572-583

39. Maurer M, Heine O, Wolf M et al (2010) Safety and tolerability of iobitridol in general and in patients with risk factors: Results in more than 160000 patients. Eur J Radiol (in press) 